\title{
Harmonic univalent functions defined by post quantum calculus operators
}

\author{
Om P. Ahuja \\ Department of Mathematics, \\ Kent State University, \\ Burton, OH, USA \\ email: oahuja@kent.edu
}

\author{
Asena Çetinkaya \\ Department of Mathematics and \\ Computer Sciences, İstanbul Kültür \\ University, İstanbul, Turkey \\ email: asnfigen@hotmail.com
}

\author{
V. Ravichandran \\ Department of Mathematics, \\ National Institute of Technology, \\ Tiruchirappalli-620015, India \\ email: ravic@nitt.edu; vravi68@gmail.com
}

\begin{abstract}
We study a family of harmonic univalent functions in the open unit disc defined by using post quantum calculus operators. We first obtained a coefficient characterization of these functions. Using this, coefficients estimates, distortion and covering theorems were also obtained. The extreme points of the family and a radius result were also obtained. The results obtained include several known results as special cases.
\end{abstract}

\section{Introduction}

Let $\mathcal{A}$ be the class of functions $\mathrm{f}$ that are analytic in the open unit disc $\mathbb{D}:=\{z:|z|<1\}$ with the normalization $\mathrm{f}(0)=\mathrm{f}^{\prime}(0)-1=0$. A function $\mathrm{f} \in \mathcal{A}$ can be expressed in the form

$$
f(z)=z+\sum_{k=2}^{\infty} a_{k} z^{k}, \quad z \in \mathbb{D} .
$$

2010 Mathematics Subject Classification: 30C50, 30C99, 81Q99

Key words and phrases: (p, q)-calculus, q-calculus, (p, q)-Sălăgean harmonic function, Sălăgean differential operator 
The theory of $(p, q)$-calculus (or post quantum calculus) operators are used in various areas of science and also in the geometric function theory. Let $0<$ $q \leq p \leq 1$. The $(p, q)$-bracket or twin-basic number $[k]_{p, q}$ is defined by

$$
[k]_{p, q}=\frac{p^{k}-q^{k}}{p-q} \quad(q \neq p), \quad \text { and } \quad[k]_{p, p}=k p^{k-1} .
$$

Notice that $\lim _{\mathrm{q} \rightarrow \mathrm{p}}[\mathrm{k}]_{\mathrm{p}, \mathrm{q}}=[\mathrm{k}]_{\mathrm{p}, \mathrm{p}}$. For $0<\mathrm{q} \leq 1$, $\mathrm{q}$-bracket $[\mathrm{k}]_{\mathrm{q}}$ for $\mathrm{k}=$ $0,1,2, \cdots$ is given by

$$
[k]_{\mathrm{q}}=[\mathrm{k}]_{1, \mathrm{q}}=\frac{1-\mathrm{q}^{\mathrm{k}}}{1-\mathrm{q}} \quad(\mathrm{q} \neq 1), \quad \text { and } \quad[\mathrm{k}]_{1}=[\mathrm{k}]_{1,1}=\mathrm{k} .
$$

The $(p, q)$-derivative operator $D_{p, q}$ of a function $f \in \mathcal{A}$ is given by

$$
D_{p, q} f(z)=1+\sum_{k=2}^{\infty}[k]_{p, q} a_{k} z^{k-1} .
$$

For a function $\mathrm{f} \in \mathcal{A}$, it can be easily seen that

$$
D_{p, q} f(z)=\frac{f(p z)-f(q z)}{(p-q) z}, \quad(p \neq q, z \neq 0),
$$

$\left(D_{p, q} f\right)(0)=1$ and $\left(D_{p, p} f\right)(z)=f^{\prime}(z)$. For definitions and properties of $(p, q)$ calculus, one may refer to $[6]$. The $(1, q)$-derivative operator $D_{1, q}$ is known as the $\mathrm{q}$-derivative operator and is denoted by $\mathrm{D}_{\mathrm{q}}$; for $z \neq 0$, it satisfies

$$
\left(D_{q} f\right)(z)=\frac{f(z)-f(q z)}{(1-q) z}
$$

For definitions and properties of q-derivative operator, one may refer to $[3,9$, $10,11,8]$.

For a function $h$ analytic in $\mathbb{D}$ and an integer $m \geq 0$, we define the $(p, q)$ Sălăgean differential operator $L_{p, q}^{m}$, using $(p, q)$-derivative operator, by

$$
\mathrm{L}_{\mathrm{p}, \mathrm{q}}^{0} \mathrm{~h}(z)=h(z) \quad \text { and } \quad \mathrm{L}_{\mathrm{p}, \mathrm{q}}^{\mathrm{m}} \mathrm{h}(z)=z \mathrm{D}_{\mathrm{p}, \mathrm{q}}\left(\mathrm{L}_{\mathrm{p}, \mathrm{q}}^{\mathrm{m}-1}(\mathrm{~h}(z))\right. \text {. }
$$

For analytic function $g(z)=\sum_{k=1}^{\infty} b_{k} z^{k}$, we have

$$
\mathrm{L}_{\mathrm{p}, \mathrm{q}}^{\mathrm{m}} \mathrm{g}(z)=\sum_{\mathrm{k}=1}^{\infty}[k]_{\mathrm{p}, \mathrm{q}}^{\mathrm{m}} \mathrm{b}_{\mathrm{k}} z^{\mathrm{k}} .
$$


In particular, for $h \in \mathcal{A}$ with $h(z)=z+\sum_{k=2}^{\infty} a_{k} z^{k}$, we have

$$
\mathrm{L}_{\mathrm{p}, \mathrm{q}}^{\mathrm{m}} \mathrm{h}(z)=z+\sum_{\mathrm{k}=2}^{\infty}[k]_{\mathrm{p}, \mathrm{q}}^{\mathrm{m}} \mathrm{a}_{\mathrm{k}} z^{\mathrm{k}} \text {. }
$$

Let $\mathcal{H}$ be the family of complex-valued harmonic functions $f=h+\bar{g}$ defined in $\mathbb{D}$, where $h$ and $g$ has the following power series expansion

$$
h(z)=z+\sum_{k=2}^{\infty} a_{k} z^{k} \quad \text { and } \quad g(z)=\sum_{k=1}^{\infty} b_{k} z^{k} .
$$

Note that $f=h+\bar{g}$ is sense-preserving in $\mathbb{D}$ if and only if $h^{\prime}(z) \neq 0$ in $\mathbb{D}$ and the second dilatation $w$ of $f$ satisfies the condition $\left|g^{\prime}(z) / h^{\prime}(z)\right|<1$ in $\mathbb{D}$. Let $\mathcal{S}_{\mathcal{H}}$ be a subclass of functions $f$ in $\mathcal{H}$ that are sense-preserving and univalent in $\mathbb{D}$. Clunie and Sheil-Small studied the class $\mathcal{S}_{\mathcal{H}}$ in their remarkable paper [5]. For a survey or comprehensive study of the theory of harmonic univalent functions, one may refer to the papers $[1,2,7]$. We introduce and study a new subclass of harmonic univalent functions by using $(p, q)$-Sălăgean harmonic differential operator $\mathrm{L}_{p, q}^{\mathrm{m}}: \mathcal{H} \rightarrow \mathcal{H}$. For the functions in the newly introduced family, a coefficient characterization is obtained (Theorem 3). Using this, coefficients estimates (Corollary 4), distortion (Theorem 6) and covering (Corollary 7) theorems were also obtained. The extreme points of the family (Theorem 5) and a radius result (Theorem 8) were also obtained. The results obtained include several known results as special cases. Our results can be extended, for example, by using fractional q-integral operator (see Ravikumar [16]).

\section{Main results}

We define the ( $p, q)$-Sălăgean harmonic differential operator $L_{p, q}^{m}$ of a harmonic function $f=h+\bar{g} \in \mathcal{H}$ by

$$
\begin{aligned}
\mathrm{L}_{\mathrm{p}, \mathrm{q}}^{\mathrm{m}} \mathrm{f}(z) & =\mathrm{L}_{\mathrm{p}, \mathrm{q}}^{\mathrm{m}} \mathrm{h}(z)+(-1)^{\mathrm{m}} \overline{\mathrm{L}_{\mathrm{p}, \mathrm{q}}^{\mathrm{m} g} \mathrm{~g}(z)} \\
& =z+\sum_{\mathrm{k}=2}^{\infty}[k]_{\mathrm{p}, \mathrm{q}}^{\mathrm{m}} \mathrm{a}_{\mathrm{k}} z^{\mathrm{k}}+(-1)^{\mathrm{m}} \sum_{\mathrm{k}=1}^{\infty}[k]_{\mathrm{p}, \mathrm{q}}^{\mathrm{m}} \overline{b_{k} z^{k}} .
\end{aligned}
$$

This last expression is obtained by using (6) and (5) and is motivated by Sălăgean[17]. Recall that convolution (or the Hadamard product) of two complexvalued harmonic functions

$$
\mathrm{f}_{1}(z)=z+\sum_{\mathrm{k}=2}^{\infty} \mathrm{a}_{1 \mathrm{k}} z^{\mathrm{k}}+\sum_{\mathrm{k}=1}^{\infty} \overline{\mathrm{b}_{1 \mathrm{k}} z^{\mathrm{k}}} \quad \text { and } \quad \mathrm{f}_{2}(z)=z+\sum_{\mathrm{k}=2}^{\infty} \mathrm{a}_{2 \mathrm{k}} z^{\mathrm{k}}+\sum_{\mathrm{k}=1}^{\infty} \overline{\mathrm{b}_{2 \mathrm{k}} z^{\mathrm{k}}}
$$


is defined by

$$
f_{1}(z) * f_{2}(z)=\left(f_{1} * f_{2}\right)(z)=z+\sum_{k=2}^{\infty} a_{1 k} a_{2 k} z^{k}+\sum_{k=1}^{\infty} \overline{b_{1 k} b_{2 k} z^{k}}, \quad z \in \mathbb{D} .
$$

We now introduce a family of ( $p, q)$-Sălăgean harmonic univalent functions by using convolution and the ( $p, q)$-Sălăgean harmonic differential operator $\mathrm{L}_{\mathrm{p}, \mathrm{q}}^{\mathrm{m}}$.

Definition 1 Suppose $i, j \in\{0,1\}$. Let the function $\Phi_{i}, \Psi_{j}$ given by

$$
\begin{aligned}
& \Phi_{i}(z)=z+\sum_{k=2}^{\infty} \lambda_{k} z^{k}+(-1)^{i} \sum_{k=1}^{\infty} \mu_{k} \bar{z}^{k}, \\
& \Psi_{j}(z)=z+\sum_{k=2}^{\infty} u_{k} z^{k}+(-1)^{j} \sum_{k=1}^{\infty} v_{k} \bar{z}^{k}
\end{aligned}
$$

be harmonic in $\mathbb{D}$ with $\lambda_{\mathrm{k}}>\mathfrak{u}_{\mathrm{k}} \geq 0(\mathrm{k} \geq 2)$ and $\mu_{\mathrm{k}}>v_{\mathrm{k}} \geq 0(\mathrm{k} \geq 1)$. For $\alpha \in[0,1), 0<\mathrm{q} \leq \mathrm{p} \leq 1, \mathrm{~m} \in \mathbb{N}, \mathrm{n} \in \mathbb{N}_{0}, \mathrm{~m}>\mathrm{n}$ and $z \in \mathbb{D}$, let $\mathcal{S}_{\mathrm{H}}\left(\mathrm{m}, \mathrm{n}, \Phi_{i}, \Psi_{j}, \mathrm{p}, \mathrm{q}, \alpha\right)$ denote the family of harmonic functions $\mathrm{f}$ in $\mathcal{H}$ that satisfy the condition

$$
\operatorname{Re}\left\{\frac{\left(L_{p, q}^{m} f * \Phi_{i}\right)(z)}{\left(L_{p, q}^{n} f * \Psi_{j}\right)(z)}\right\}>\alpha,
$$

where $\mathrm{L}_{\mathbf{p}, \mathbf{q}}^{\mathrm{m}}$ is defined by (8).

Using (8), (9) and (10), we obtain

$$
\left(L_{p, q}^{m} f * \Phi_{i}\right)(z)=z+\sum_{k=2}^{\infty} \lambda_{k}[k]_{p, q}^{m} a_{k} z^{k}+(-1)^{m+i} \sum_{k=1}^{\infty} \mu_{k}[k]_{p, q}^{m} b_{k} \bar{z}^{k},
$$

and

$$
\left(\mathrm{L}_{\mathrm{p}, \mathrm{q}}^{\mathrm{n}} \mathrm{f} * \Psi_{j}\right)(z)=z+\sum_{k=2}^{\infty} u_{k}[k]_{p, q}^{n} a_{k} z^{k}+(-1)^{n+j} \sum_{k=1}^{\infty} v_{k}[k]_{p, q}^{n} b_{k} \bar{z}^{k} .
$$

Definition 2 Let $\mathcal{T} \mathcal{S}_{\mathrm{H}}\left(\mathrm{m}, \mathrm{n}, \Phi_{i}, \Psi_{j}, \mathrm{p}, \mathrm{q}, \alpha\right)$ be the family of harmonic functions $\mathrm{f}_{\mathrm{m}}=\mathrm{h}+\bar{g}_{\mathrm{m}} \in \mathcal{T} \mathcal{S}_{\mathrm{H}}\left(\mathrm{m}, \mathrm{n}, \Phi_{i}, \Psi_{j}, \mathrm{p}, \mathrm{q}, \alpha\right)$ such that $\mathrm{h}$ and $\mathrm{g}_{\mathrm{m}}$ are of the form

$$
h(z)=z-\sum_{k=2}^{\infty}\left|a_{k}\right| z^{k} \quad \text { and } \quad g_{m}(z)=(-1)^{m+i-1} \sum_{k=1}^{\infty}\left|b_{k}\right| z^{k}, \quad\left|b_{1}\right|<1 .
$$


The families of $\mathcal{S}_{\mathrm{H}}\left(\mathrm{m}, \mathrm{n}, \Phi_{i}, \Psi_{j}, \mathrm{p}, \mathrm{q}, \alpha\right)$ and $\mathcal{T} \mathcal{S}_{\mathrm{H}}\left(\mathrm{m}, \mathrm{n}, \Phi_{i}, \Psi_{j}, \mathrm{p}, \mathrm{q}, \alpha\right)$ include a variety of well-known subclasses of harmonic functions as well as many new ones. For example,

(1) $\mathcal{S}_{\mathrm{H}}(\mathrm{m}, \mathrm{n}, \alpha) \equiv \mathcal{S}_{\mathrm{H}}\left(\mathrm{m}, \mathrm{n}, \frac{z}{(1-z)^{2}}-\frac{\bar{z}}{(1-\bar{z})^{2}}, \frac{z}{1-z}+\frac{\bar{z}}{1-\bar{z}}, 1,1, \alpha\right)$, $\mathcal{T} \mathcal{S}_{\mathrm{H}}(\mathrm{m}, \mathrm{n}, \alpha) \equiv \mathcal{T} \mathcal{S}_{\mathrm{H}}\left(\mathrm{m}, \mathrm{n}, \frac{z}{(1-z)^{2}}-\frac{\bar{z}}{(1-\bar{z})^{2}}, \frac{z}{1-z}+\frac{\bar{z}}{1-\bar{z}}, 1,1, \alpha\right),[18]$.

(2) $\mathcal{S}_{\mathrm{H}}^{*}(\alpha) \equiv \mathcal{S}_{\mathrm{H}}\left(1,0, \frac{z}{(1-z)^{2}}-\frac{\bar{z}}{(1-\bar{z})^{2}}, \frac{z}{1-z}+\frac{\bar{z}}{1-\bar{z}}, 1,1, \alpha\right)$, $\mathcal{T S}_{\mathrm{H}}^{*}(\alpha) \equiv \mathcal{T} \mathcal{S}_{\mathrm{H}}\left(1,0, \frac{z}{(1-z)^{2}}-\frac{\bar{z}}{(1-\bar{z})^{2}}, \frac{z}{1-z}+\frac{\bar{z}}{1-\bar{z}}, 1,1, \alpha\right),[12]$.

(3) $\mathcal{K}_{\mathrm{H}}(\alpha) \equiv \mathcal{S}_{\mathrm{H}}\left(2,1, \frac{z+z^{2}}{(1-z)^{3}}+\frac{\bar{z}+\bar{z}^{2}}{(1-\bar{z})^{3}}, \frac{z}{(1-z)^{2}}-\frac{\bar{z}}{(1-\bar{z})^{2}}, 1,1, \alpha\right)$, $\mathcal{T} \mathcal{K}_{\mathrm{H}}(\alpha) \equiv \mathcal{T} \mathcal{S}_{\mathrm{H}}\left(2,1, \frac{z+z^{2}}{(1-z)^{3}}+\frac{\bar{z}+\bar{z}^{2}}{(1-\bar{z})^{3}}, \frac{z}{(1-z)^{2}}-\frac{\bar{z}}{(1-\bar{z})^{2}}, 1,1, \alpha\right),[13]$.

(4) $\mathcal{S}_{\mathrm{H}_{\mathrm{q}}}^{*}(\alpha) \equiv \mathcal{S}_{\mathrm{H}}\left(1,0, \frac{z}{(1-z)^{2}}-\frac{\bar{z}}{(1-\bar{z})^{2}}, \frac{z}{1-z}+\frac{\bar{z}}{1-\bar{z}}, 1, \mathrm{q}, \alpha\right)$, $\mathcal{T S}_{\mathrm{H}_{\mathrm{q}}}^{*}(\alpha) \equiv \mathcal{T} \mathcal{S}_{\mathrm{H}}\left(1,0, \frac{z}{(1-z)^{2}}-\frac{\bar{z}}{(1-\bar{z})^{2}}, \frac{z}{1-z}+\frac{\bar{z}}{1-\bar{z}}, 1, \mathbf{q}, \alpha\right),[4]$.

(5) $\mathcal{K}_{\mathrm{H}_{\mathrm{q}}}(\alpha) \equiv \mathcal{S}_{\mathrm{H}}\left(2,1, \frac{z+z^{2}}{(1-z)^{3}}+\frac{\bar{z}+\bar{z}^{2}}{(1-\bar{z})^{3}}, \frac{z}{(1-z)^{2}}-\frac{\bar{z}}{(1-\bar{z})^{2}}, 1, \mathrm{q}, \alpha\right)$, $\mathcal{T} \mathcal{K}_{\mathrm{H}_{\mathrm{q}}}(\alpha) \equiv \mathcal{T} \mathcal{S}_{\mathrm{H}}\left(2,1, \frac{z+z^{2}}{(1-z)^{3}}+\frac{\bar{z}+\bar{z}^{2}}{(1-\bar{z})^{3}}, \frac{z}{(1-z)^{2}}-\frac{\bar{z}}{(1-\bar{z})^{2}}, 1, \mathrm{q}, \alpha\right)$.

(6) $\mathcal{S}_{\mathrm{H}}(\mathrm{n}+1, \mathrm{n}, \mathrm{q}, \alpha) \equiv \mathcal{S}_{\mathrm{H}}\left(\mathrm{n}+1, \mathrm{n}, \frac{z}{(1-z)^{2}}-\frac{\bar{z}}{(1-\bar{z})^{2}}, \frac{z}{1-z}+\frac{\bar{z}}{1-\bar{z}}, 1, \mathrm{q}, \alpha\right)$, $\mathcal{T} \mathcal{S}_{\mathrm{H}}(\mathrm{n}+1, \mathrm{n}, \mathrm{q}, \alpha) \equiv \mathcal{T} \mathcal{S}_{\mathrm{H}}\left(\mathrm{n}+1, \mathrm{n}, \frac{z}{(1-z)^{2}}-\frac{\bar{z}}{(1-\bar{z})^{2}}, \frac{z}{1-z}+\frac{\bar{z}}{1-\bar{z}}, 1, \mathrm{q}, \alpha\right)$, [14].

(7) $\mathcal{S}_{\mathrm{H}}\left(\Phi_{i}, \Psi_{j}, \alpha\right) \equiv \mathcal{S}_{\mathrm{H}}\left(0,0, \Phi_{i}, \Psi_{j}, 1,1, \alpha\right)$ $\mathcal{T} \mathcal{S}_{\mathrm{H}}\left(\Phi_{i}, \Psi_{j}, \alpha\right) \equiv \mathcal{T} \mathcal{S}_{\mathrm{H}}\left(0,0, \Phi_{i}, \Psi_{j}, 1,1, \alpha\right),[15]$.

We first prove coefficient conditions for the functions in $\mathcal{S}_{H}\left(m, n, \Phi_{i}, \Psi_{j}, p, q, \alpha\right)$ and $\mathcal{T} \mathcal{S}_{\mathrm{H}}\left(\mathrm{m}, \mathrm{n}, \Phi_{i}, \Psi_{j}, \mathrm{p}, \mathrm{q}, \alpha\right)$.

Theorem 3 Let the function $\mathrm{f}=\mathrm{h}+\overline{\mathrm{g}}$ be such that the functions $\mathrm{h}$ and $\mathrm{g}$ are given by (7). Also, let the (p, q)-coefficient inequality

$$
\begin{aligned}
& \sum_{k=2}^{\infty} \frac{\lambda_{k}[k]_{p, q}^{m}-\alpha u_{k}[k]_{p, q}^{n}}{1-\alpha}\left|a_{k}\right| \\
& +\sum_{k=1}^{\infty} \frac{\mu_{k}[k]_{p, q}^{m}-(-1)^{n+j-(m+i)} \alpha v_{k}[k]_{p, q}^{n}}{1-\alpha}\left|b_{k}\right| \leq 1,
\end{aligned}
$$

be satisfied for $\alpha \in[0,1), 0<\mathrm{q} \leq \mathrm{p} \leq 1, \mathrm{~m} \in \mathbb{N}, \mathrm{n} \in \mathbb{N}_{0}, \mathrm{~m}>\mathrm{n}, \lambda_{\mathrm{k}}>\mathfrak{u}_{\mathrm{k}} \geq 0$ $(k \geq 2)$ and $\mu_{k}>v_{k} \geq 0(k \geq 1)$. Then 
(i) the function $\mathrm{f}=\mathrm{h}+\overline{\mathrm{g}}$ given by (7) is a sense-preserving harmonic univalent functions in $\mathbb{D}$ and $\mathrm{f} \in \mathcal{S}_{\mathrm{H}}\left(\mathrm{m}, \mathrm{n}, \Phi_{i}, \Psi_{j}, \mathrm{p}, \mathrm{q}, \alpha\right)$ if the inequality in (15) is satisfied.

(ii) the function $\mathrm{f}_{\mathrm{m}}=\mathrm{h}+\overline{\mathrm{g}}_{\mathrm{m}}$ given by (14) is in the $\mathcal{T} \mathcal{S}_{\mathrm{H}}\left(\mathrm{m}, \mathrm{n}, \Phi_{\mathfrak{i}}, \Psi_{\mathbf{j}}, \mathrm{p}, \mathrm{q}, \alpha\right)$ if and only if the inequality in (15) is satisfied.

Proof. (i). Using the techniques used in [14] and [15], it is a routine step to prove that $f=h+\bar{g}$ given by (7) is sense-preserving and locally univalent in $\mathbb{D}$. Using the fact $\operatorname{Re}(w)>\alpha$ if and only if $|1-\alpha+w| \geq|1+\alpha-w|$, it suffices to show that

$$
\left|1-\alpha+\frac{\left(L_{p, q}^{m} f * \Phi_{i}\right)(z)}{\left(L_{p, q}^{n} f * \Psi_{j}\right)(z)}\right|-\left|1+\alpha-\frac{\left(L_{p, q}^{m} f * \Phi_{i}\right)(z)}{\left(L_{p, q}^{n} f * \Psi_{j}\right)(z)}\right| \geq 0 .
$$

In view of (12) and (13), left side of (16) yields

$$
\begin{aligned}
& \left|\left(L_{p, q}^{m} f * \Phi_{i}\right)(z)+(1-\alpha)\left(L_{p, q}^{n} f * \Psi_{j}\right)(z)\right| \\
& -\left|\left(L_{p, q}^{m} f * \Phi_{i}\right)(z)-(1+\alpha)\left(L_{p, q}^{n} f * \Psi_{j}\right)(z)\right| \\
& =\mid(2-\alpha) z+\sum_{k=2}^{\infty}\left(\lambda_{k}[k]_{p, q}^{m}+(1-\alpha) u_{k}[k]_{p, q}^{n}\right) a_{k} z^{k} \\
& +(-1)^{m+i} \sum_{k=1}^{\infty}\left(\mu_{k}[k]_{p, q}^{m}+(-1)^{n+j-(m+i)}(1-\alpha) v_{k}[k]_{p, q}^{n}\right) b_{k} \bar{z}^{k} \\
& -\mid-\alpha z+\sum_{k=2}^{\infty}\left(\lambda_{k}[k]_{p, q}^{m}-(1+\alpha) u_{k}[k]_{p, q}^{n}\right) a_{k} z^{k} \\
& +(-1)^{m+i} \sum_{k=1}^{\infty}\left(\mu_{k}[k]_{p, q}^{m}-(-1)^{n+j-(m+i)}(1+\alpha) v_{k}[k]_{p, q}^{n}\right) b_{k} \bar{z}^{k} \\
& \geq(2-2 \alpha)|z|-2 \sum_{k=2}^{\infty}\left(\lambda_{k}[k]_{p, q}^{m}-\alpha u_{k}[k]_{p, q}^{n}\right)\left|a_{k}\right||z|^{k} \\
& -\sum_{k=1}^{\infty}\left(\mu_{k}[k]_{p, q}^{m}+(-1)^{n+j-(m+i)}(1-\alpha) v_{k}[k]_{p, q}^{n}\right)|b|_{k}|z|^{k} \\
& -\sum_{k=1}^{\infty}\left(\mu_{k}[k]_{p, q}^{m}-(-1)^{n+j-(m+i)}(1+\alpha) v_{k}[k]_{p, q}^{n}\right)|b|_{k}|z|^{k} \\
& \geq(1-\alpha)|z|\left[1-\sum_{k=2}^{\infty} \frac{\lambda_{k}[k]_{p, q}^{m}-\alpha u_{k}[k]_{p, q}^{n}}{1-\alpha}\left|a_{k}\right||z|^{k-1}\right.
\end{aligned}
$$




$$
\begin{aligned}
& \left.-\sum_{k=1}^{\infty} \frac{\mu_{k}[k]_{p, q}^{m}-(-1)^{n+j-(m+i)} \alpha v_{k}[k]_{p, q}^{n}}{1-\alpha}\left|b_{k}\right||z|^{k-1}\right] \\
> & (1-\alpha)|z|\left[1-\left(\sum_{k=2}^{\infty} \frac{\lambda_{k}[k]_{p, q}^{m}-\alpha u_{k}[k]_{p, q}^{n}}{1-\alpha}\left|a_{k}\right|\right.\right. \\
& \left.\left.+\sum_{k=1}^{\infty} \frac{\mu_{k}[k]_{p, q}^{m}-(-1)^{n+j-(m+i)} \alpha v_{k}[k]_{p, q}^{n}}{1-\alpha}\left|b_{k}\right|\right)\right] .
\end{aligned}
$$

This last expression is non-negative because of the condition given in (15). This completes the proof of part (i) of theorem.

(ii). Since

$$
\mathcal{T} \mathcal{S}_{\mathrm{H}}\left(\mathrm{m}, \mathrm{n}, \Phi_{i}, \Psi_{j}, p, \mathrm{q}, \alpha\right) \subset \mathcal{S}_{\mathrm{H}}\left(\mathrm{m}, \mathrm{n}, \Phi_{i}, \Psi_{j}, \mathrm{p}, \mathrm{q}, \alpha\right),
$$

the sufficient part of part (ii) follows from part (i). In order to prove the

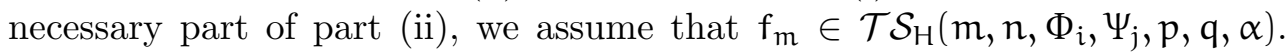
We notice that

$$
\begin{aligned}
& \operatorname{Re}\left\{\frac{\left(L_{p, q}^{m} f * \Phi_{i}\right)(z)}{\left(L_{p, q}^{n} f * \Psi_{j}\right)(z)}-\alpha\right\} \\
& =\operatorname{Re}\left\{\frac{(1-\alpha) z-\sum_{k=2}^{\infty}\left(\lambda_{k}[k]_{p, q}^{m}-\alpha u_{k}[k]_{p, q}^{n}\right) a_{k} z^{k}}{z-\sum_{k=2}^{\infty} u_{k}[k]_{p, q}^{n} a_{k} z^{k}+(-1)^{m+i+n+j-1} \sum_{k=1}^{\infty} v_{k}[k]_{p, q}^{n} b_{k} \bar{z}^{k}}\right. \\
& \left.+\frac{(-1)^{2 m+2 i-1} \sum_{k=1}^{\infty}\left(\mu_{k}[k]_{p, q}^{m}-(-1)^{n+j-(m+i)} \alpha v_{k}[k]_{p, q}^{n}\right) b_{k} \bar{z}^{k}}{z-\sum_{k=2}^{\infty} u_{k}[k]_{p, q}^{n} a_{k} z^{k}+(-1)^{m+i+n+j-1} \sum_{k=1}^{\infty} v_{k}[k]_{p, q}^{n} b_{k} \bar{z}^{k}}\right\} \\
& \geq \frac{(1-\alpha)-\sum_{k=2}^{\infty}\left(\lambda_{k}[k]_{p, q}^{m}-\alpha u_{k}[k]_{p, q}^{n}\right) a_{k} r^{k-1}}{1-\sum_{k=2}^{\infty} u_{k}[k]_{p, q}^{n} a_{k} r^{k-1}-(-1)^{m+i+n+j} \sum_{k=1}^{\infty} v_{k}[k]_{p, q}^{n} b_{k} r^{k-1}} \\
& \geq 0,
\end{aligned}
$$

by (11). The above inequality must hold for all $z \in \mathbb{D}$. In particular, choosing the values of $z$ on the positive real axis and $z \rightarrow 1^{-}$, we obtain the required condition (15). This completes the proof of part (ii) of theorem. 
The harmonic mappings

$$
\begin{aligned}
f(z)= & z+\sum_{k=2}^{\infty} \frac{1-\alpha}{\lambda_{k}[k]_{p, q}^{m}-\alpha u_{k}[k]_{p, q}^{n}} x_{k} z^{k} \\
& +\sum_{k=1}^{\infty} \frac{1-\alpha}{\mu_{k}[k]_{p, q}^{m}-(-1)^{n+j-(m+i)} \alpha v_{k}[k]_{p, q}^{n}} y_{k} \bar{z}^{k}
\end{aligned}
$$

where $\sum_{k=2}^{\infty}\left|x_{k}\right|+\sum_{k=1}^{\infty}\left|y_{k}\right|=1$, show that the coefficient bound given by (15) is sharp.

Theorem 3 also yields the following corollary.

Corollary 4 For the function $\mathrm{f}_{\mathrm{m}}=\mathrm{h}+\overline{\mathrm{g}}_{\mathrm{m}}$ given by (14), we have

$$
\left|a_{k}\right| \leq \frac{1-\alpha}{\lambda_{k}[k]_{p, q}^{m}-\alpha u_{k}[k]_{p, q}^{n}}, \quad k \geq 2
$$

and

$$
\left|b_{k}\right| \leq \frac{1-\alpha}{\mu_{k}[k]_{p, q}^{m}-(-1)^{n+j-(m+i)} \alpha v_{k}[k]_{p, q}^{n}}, \quad k \geq 1 .
$$

The result is sharp for each $\mathrm{k}$.

Using Theorem 3 (part ii), it is seen that the class $\mathcal{T} \mathcal{S}_{\mathrm{H}}\left(\mathrm{m}, \mathrm{n}, \Phi_{i}, \Psi_{j}, \mathrm{p}, \mathrm{q}, \alpha\right)$ is convex and closed with respect to the topology of locally uniform convergence so that the closed convex hulls of $\mathcal{T} \mathcal{S}_{\mathrm{H}}\left(\mathrm{m}, \mathrm{n}, \Phi_{i}, \Psi_{j}, \mathrm{p}, \mathrm{q}, \alpha\right)$ equals itself. The next theorem determines the extreme points of $\mathcal{T} \mathcal{S}_{\mathrm{H}}\left(\mathrm{m}, \mathrm{n}, \Phi_{\mathrm{i}}, \Psi_{\mathrm{j}}, \mathrm{p}, \mathrm{q}, \alpha\right)$.

Theorem 5 Let the function $\mathrm{f}_{\mathrm{m}}=\mathrm{h}+\overline{\mathrm{g}}_{\mathrm{m}}$ be given by (14). Then the function $\mathrm{f}_{\mathrm{m}} \in \operatorname{clco} \mathcal{T} \mathcal{S}_{\mathrm{H}}\left(\mathrm{m}, \mathrm{n}, \Phi_{\mathrm{i}}, \Psi_{\mathrm{j}}, \mathrm{p}, \mathrm{q}, \alpha\right)$ if and only if $\mathrm{f}_{\mathrm{m}}(z)=\sum_{\mathrm{k}=1}^{\infty}\left(x_{\mathrm{k}} \mathrm{h}_{\mathrm{k}}(z)+\right.$ $\left.\mathrm{y}_{\mathrm{k}} \mathrm{g}_{\mathrm{m}_{\mathrm{k}}}(z)\right)$, where

$$
\begin{gathered}
h_{1}(z)=z, \quad h_{k}(z)=z-\frac{1-\alpha}{\lambda_{k}[k]_{p, q}^{m}-\alpha u_{k}[k]_{\mathfrak{p}, q}^{n}} z^{k}, \quad k \geq 2, \\
g_{m_{k}}(z)=z+(-1)^{m+i-1} \frac{1-\alpha}{\mu_{k}[k]_{p, q}^{m}-(-1)^{n+j-(m+i)} \alpha v_{k}[k]_{p, q}^{n}} \bar{z}^{k}, \quad k \geq 1,
\end{gathered}
$$

and $\sum_{\mathrm{k}=1}^{\infty}\left(\mathrm{x}_{\mathrm{k}}+\mathrm{y}_{\mathrm{k}}\right)=1$ where $\mathrm{x}_{\mathrm{k}} \geq 0$ and $\mathrm{y}_{\mathrm{k}} \geq 0$. In particular, the extreme points of $\mathcal{T} \mathcal{S}_{\mathrm{H}}\left(\mathrm{m}, \mathrm{n}, \Phi_{i}, \Psi_{j}, \mathrm{p}, \mathrm{q}, \alpha\right)$ are $\left\{\mathrm{h}_{\mathrm{k}}\right\}$ and $\left\{\mathrm{g}_{\mathrm{m}_{\mathrm{k}}}\right\}$. 
Proof. For a function $f_{m}$ of the form $f_{m}(z)=\sum_{k=1}^{\infty}\left(x_{k} h_{k}(z)+y_{k} g_{m_{k}}(z)\right)$, where $\sum_{k=1}^{\infty}\left(x_{k}+y_{k}\right)=1$, we have

$$
\begin{aligned}
f_{m}(z)= & z-\sum_{k=2}^{\infty} \frac{1-\alpha}{\lambda_{k}[k]_{p, q}^{m}-\alpha u_{k}[k]_{p, q}^{n}} x_{k} z^{k} \\
& +\sum_{k=1}^{\infty}(-1)^{m+i-1} \frac{1-\alpha}{\mu_{k}[k]_{p, q}^{m}-(-1)^{n+j-(m+i)} \alpha v_{k}[k]_{p, q}^{n}} y_{k} \bar{z}^{k}
\end{aligned}
$$

Then $\mathrm{f}_{\mathrm{m}} \in \operatorname{clco} \mathcal{T} \mathcal{S}_{\mathrm{H}}\left(\mathrm{m}, \mathrm{n}, \Phi_{i}, \Psi_{j}, p, \mathrm{q}, \alpha\right)$ because

$$
\begin{gathered}
\sum_{k=2}^{\infty} \frac{\lambda_{k}[k]_{p, q}^{m}-\alpha u_{k}[k]_{p, q}^{n}}{1-\alpha}\left(\frac{1-\alpha}{\lambda_{k}[k]_{p, q}^{m}-\alpha u_{k}[k]_{p, q}^{n}} x_{k}\right)+ \\
\sum_{k=1}^{\infty} \frac{\mu_{k}[k]_{p, q}^{m}-(-1)^{n+j-(m+i)} \alpha v_{k}[k]_{p, q}^{n}}{1-\alpha}\left(\frac{1-\alpha}{\mu_{k}[k]_{p, q}^{m}-(-1)^{n+j-(m+i)} \alpha v_{k}[k]_{p, q}^{n}} y_{k}\right) \\
=\sum_{k=2}^{\infty} x_{k}+\sum_{k=1}^{\infty} y_{k}=1-x_{1} \leq 1 .
\end{gathered}
$$

Conversely, suppose $\mathrm{f}_{\mathrm{m}} \in \operatorname{clco} \mathcal{T} \mathcal{S}_{\mathrm{H}}\left(\mathrm{m}, \mathrm{n}, \Phi_{i}, \Psi_{\mathrm{j}}, \mathrm{p}, \mathrm{q}, \alpha\right)$. Then

$$
\left|a_{k}\right| \leq \frac{1-\alpha}{\lambda_{k}[k]_{p, q}^{m}-\alpha u_{k}[k]_{p, q}^{n}} \quad \text { and } \quad\left|b_{k}\right| \leq \frac{1-\alpha}{\mu_{k}[k]_{p, q}^{m}-(-1)^{n+j-(m+i)} \alpha v_{k}[k]_{p, q}^{n}}
$$

Set

$x_{k}=\frac{\lambda_{k}[k]_{p, q}^{m}-\alpha u_{k}[k]_{p, q}^{n}}{1-\alpha}\left|a_{k}\right|$ and $y_{k}=\frac{\mu_{k}[k]_{p, q}^{m}-(-1)^{n+j-(m+i)} \alpha v_{k}[k]_{p, q}^{n}}{1-\alpha}\left|b_{k}\right|$.

By Theorem 3 (ii), $\sum_{k=2}^{\infty} x_{k}+\sum_{k=1}^{\infty} y_{k} \leq 1$. Therefore we define $x_{1}=1-\sum_{k=2}^{\infty} x_{k}-$ $\sum_{k=1}^{\infty} y_{k} \geq 0$. Consequently, we obtain $f_{m}(z)=\sum_{k=1}^{\infty}\left(x_{k} h_{k}(z)+y_{k} g_{m_{k}}(z)\right)$ as required.

For functions in the class $\mathcal{T} \mathcal{S}_{H}\left(m, n, \Phi_{i}, \Psi_{j}, p, q, \alpha\right)$, the following theorem gives distortion bounds which in turns yields the covering result for this class. 
Theorem 6 Let the function $\mathrm{f}_{\mathrm{m}} \in \mathcal{T} \mathcal{S}_{\mathrm{H}}\left(\mathrm{m}, \mathrm{n}, \Phi_{i}, \Psi_{\mathrm{j}}, \mathrm{p}, \mathrm{q}, \alpha\right), \gamma_{\mathrm{k}}=\lambda_{\mathrm{k}}[\mathrm{k}]_{\mathrm{p}, \mathrm{q}}^{\mathrm{m}}-$ $\alpha u_{k}[k]_{p, q}^{n}, k \geq 2$ and $\phi_{k}=\mu_{k}[k]_{p, q}^{m}-(-1)^{n+j-(m+i)} \alpha v_{k}[k]_{p, q}^{n}, k \geq 1$. If $\left\{\gamma_{k}\right\}$ and $\left\{\phi_{\mathrm{k}}\right\}$ are non-decreasing sequences, then we have

$$
\left|f_{m}(z)\right| \leq\left(1+\left|b_{1}\right|\right)|z|+\frac{1-\alpha}{\beta}\left(1-\frac{\mu_{1}-(-1)^{n+j-(m+i)} \alpha v_{1}}{\beta}\left|b_{1}\right|\right)|z|^{2}
$$

and

$$
\left|f_{m}(z)\right| \geq\left(1-\left|b_{1}\right|\right)|z|-\frac{1-\alpha}{\beta}\left(1-\frac{\mu_{1}-(-1)^{n+j-(m+i)} \alpha v_{1}}{\beta}\left|b_{1}\right|\right)|z|^{2},
$$

for all $z \in \mathbb{D}$, where $\mathrm{b}_{1}=\mathrm{f}_{\bar{z}}(0)$ and $\beta=\min \left\{\gamma_{2}, \phi_{2}\right\}=\min \left\{\lambda_{2}[2]_{\mathfrak{p}, q}^{m}-\alpha u_{2}[2]_{p, q}^{n}, \mu_{2}[2]_{p, q}^{m}-(-1)^{\mathfrak{m}+j-(m+\mathfrak{i})} \alpha v_{2}[2]_{p, q}^{n}\right\}$.

Proof. Let the function $f_{m} \in \mathcal{T S}_{\mathcal{H}}\left(\mathrm{m}, \mathrm{n}, \Phi_{i}, \Psi_{j}, p, q, \alpha\right)$. Taking the absolute value of $f_{m}$, we obtain

$$
\begin{aligned}
\left|f_{m}(z)\right| \leq & \left(1+\left|b_{1}\right|\right)|z|+\sum_{k=2}^{\infty}\left(\left|a_{k}\right|+\left|b_{k}\right|\right)|z|^{k} \\
\leq & \left(1+\left|b_{1}\right|\right)|z|+\sum_{k=2}^{\infty}\left(\left|a_{k}\right|+\left|b_{k}\right|\right)|z|^{2} \\
\leq & \left(1+\left|b_{1}\right|\right)|z|+\frac{1-\alpha}{\beta} \sum_{n=2}^{\infty}\left(\frac{\beta}{1-\alpha}\left|a_{k}\right|+\frac{\beta}{1-\alpha}\left|b_{k}\right|\right)|z|^{2} \\
\leq & \left(1+\left|b_{1}\right|\right)|z|+\frac{1-\alpha}{\beta} \sum_{k=2}^{\infty}\left(\frac{\lambda_{k}[k]_{p, q}^{m}-\alpha u_{k}[k]_{p, q}^{n}}{1-\alpha}\left|a_{k}\right|\right. \\
& \left.\quad+\frac{\mu_{k}[k]_{p, q}^{m}-(-1)^{n+j-(m+i)} \alpha v_{k}[k]_{p, q}^{n}}{1-\alpha}\left|b_{k}\right|\right)|z|^{2} \\
\leq & \left(1+\left|b_{1}\right|\right)|z|+\frac{1-\alpha}{\beta}\left(1-\frac{\mu_{1}-(-1)^{n+j-(m+i)} \alpha v_{1}}{1-\alpha}\left|b_{1}\right|\right)|z|^{2} .
\end{aligned}
$$

This proves (18). The proof of (19) is omitted as it is similar to the proof of (18).

The following covering result follows from the inequality (19).

Corollary 7 Under the hypothesis of Theorem 6, we have

$$
\left\{w:|w|<\frac{1}{\beta}\left(\beta-1+\alpha+\left(\mu_{1}-(-1)^{n+j-(m+i)} \alpha v_{1}-\beta\right)\left|b_{1}\right|\right)\right\} \subset f(\mathbb{D}) .
$$


Theorem 8 If the function $\mathrm{f}_{\mathrm{m}} \in \mathcal{T} \mathcal{S}_{\mathrm{H}}\left(\mathrm{m}, \mathrm{n}, \Phi_{i}, \Psi_{\mathrm{j}}, \mathrm{p}, \mathrm{q}, \alpha\right)$, then the function $\mathrm{f}_{\mathrm{m}}$ is convex in the disc

$$
|z| \leq \min _{k}\left\{\frac{1-b_{1}}{k\left[1-\frac{\mu_{1}-(-1)^{n+j-(m+i)} \alpha \nu_{1}}{1-\alpha} b_{1}\right]}\right\}^{\frac{1}{k-1}}, \quad k \geq 2 .
$$

Proof. Let $\mathrm{f}_{\mathrm{m}} \in \mathcal{T} \mathcal{S}_{\mathrm{H}}\left(\mathrm{m}, \mathrm{n}, \Phi_{i}, \Psi_{j}, p, q, \alpha\right)$ and let $\mathrm{r}, 0<\mathrm{r}<1$, be fixed. Then $\mathrm{r}^{-1} \mathrm{f}_{\mathrm{m}}(\mathrm{rz}) \in \mathcal{T} \mathcal{S}_{\mathrm{H}}\left(\mathrm{m}, \mathrm{n}, \Phi_{i}, \mathrm{p}, \mathrm{q}, \alpha\right)$ and we have

$$
\begin{aligned}
& \sum_{k=2}^{\infty} k^{2}\left(\left|a_{k}\right|+\left|b_{k}\right|\right)=\sum_{k=2}^{\infty} k\left(\left|a_{k}\right|+\left|b_{k}\right|\right) k r^{k-1} \\
& \leq \sum_{k=2}^{\infty}\left(\frac{\lambda_{k}[k]_{p, q}^{m}-\alpha u_{k}[k]_{p, q}^{n}}{1-\alpha}\left|a_{k}\right|+\frac{\mu_{k}[k]_{p, q}^{m}-(-1)^{n+j-(m+i)} \alpha v_{k}[k]_{p, q}^{n}}{1-\alpha}\left|b_{k}\right|\right) k r^{k-1} \\
& \leq \sum_{k=2}^{\infty}\left(1-\frac{\mu_{1}-(-1)^{n+j-(m+i)} \alpha v_{1}}{1-\alpha}\left|b_{1}\right|\right) k r^{k-1} \\
& \leq 1-b_{1}
\end{aligned}
$$

provided

$$
\mathrm{kr}^{\mathrm{k}-1} \leq \frac{1-\mathrm{b}_{1}}{1-\frac{\mu_{1}-(-1)^{\mathrm{n}+\mathrm{j}-(\mathrm{m}+\mathrm{i})} \alpha v_{1}}{1-\alpha} \mathrm{b}_{1}}
$$

which is true if

$$
r \leq \min _{k}\left\{\frac{1-b_{1}}{k\left[1-\frac{\mu_{1}-(-1)^{n+j-(m+i)} \alpha v_{1}}{1-\alpha} b_{1}\right]}\right\}^{\frac{1}{k-1}}, \quad k \geq 2 .
$$

Remark 9 Our results naturally includes several results known for those subclasses of harmonic functions listed after Definition 2.

\section{Acknowledgement}

The authors are thankful to the referee for useful comments. 


\section{References}

[1] O. P. Ahuja, Planar harmonic univalent and related mappings, J. Inequal. Pure Appl. Math., 6 (2005) (4), Art. 122, 18 pp.

[2] O. P. Ahuja, Recent advances in the theory of harmonic univalent mappings in the plane, Math. Student, 83 (1-4) (2014), 125-154.

[3] O. P. Ahuja, A. Çetinkaya, Y. Polatog̃lu,Bieberbach-de Branges and Fekete-Szegö inequalities for certain families of q-convex and q-close-toconvex functions, J. Comput. Anal. Appl., 26 (4) (2019), 639-649.

[4] O. P. Ahuja, A. Çetinkaya, Connecting quantum calculus and harmonic starlike functions, preprint, 2018.

[5] J. Clunie, T. Sheil-Small, Harmonic univalent functions, Ann. Acad. Sci. Fenn. Ser. A I Math., 9 (1984), 3-25.

[6] R. Jagannathan, K. Srivanasava, Two-parameter quantum algebras, twin-basic numbers, and associated generalized hypergeometric series, arXiv:math/0602613v1 [math.NT] 27 Feb 2006.

[7] P. Duren, Harmonic Mappings in the Plane, Cambridge Tracts in Mathematics, 156, Cambridge University Press, Cambridge, 2004.

[8] M. E. H. Ismail, E. Merkes, D. Styer, A generalization of starlike functions, Complex Var. Theory Appl., 14 (1-4) (1990), 77-84.

[9] F. H. Jackson, On q-functions and a certain difference operator, Trans. R. Soc. Edinburg, 46 (part II) (11) (1909), 253-281.

[10] F. H. Jackson, On q-definite integrals, Quart. J. Pure Appl. Math., 41 (1910), 193-203.

[11] F. H. Jackson, q-Difference equations, Amer. J. Math., 32 (4) (1910), 305-314.

[12] J. M. Jahangiri, Harmonic functions starlike in the unit disk, J. Math. Anal. Appl., 235 (3) (1999), 470-477.

[13] J. M. Jahangiri, Coefficient bounds and univalence criteria for harmonic functions with negative coefficients, Ann. Univ. Mariae Curie-Sktodowska Sect. A, 52 (2) (1998), 57-66. 
[14] J. M. Jahangiri, Harmonic univalent functions defined by q-calculus operators, Int. J. Math. Anal. Appl., 5 (2) (2018), 39-43.

[15] S. Nagpal and V. Ravichandran, A comprehensive class of harmonic functions defined by convolution and its connection with integral transforms and hypergeometric functions, Stud. Univ. Babeş-Bolyai Math., 59 (1) (2014), 41-55.

[16] N. Ravikumar, Certain classes of analytic functions defined by fractional q-calculus operator, Acta Univ. Sapientiae Math., 10 (1) (2018), 178-188.

[17] G. S. Sălăgean, Subclasses of univalent functions, in Complex AnalysisFifth Romanian-Finnish Seminar, Part 1 (Bucharest, 1981), 362-372, Lecture Notes in Math., 1013, Springer, Berlin.

[18] S. Yalçin, A new class of Salagean-type harmonic univalent functions, Appl. Math. Lett., 18 (2) (2005), 191-198. 